\title{
Millimeter Wave Phased Array Antenna Synthesis Using a Machine Learning Technique for Different 5G Applications
}

\author{
Shadi Danesh, Ali Araghi, Mohsen Khalily, Pei Xiao, and Rahim Tafazolli \\ Institute for Communication System(ICS), Home of the 5G Innovation Center (5GIC) \\ University of Surrey, Guildford, GU2 7XH, UK.
}

\begin{abstract}
A machine learning (ML) technique has been used to synthesis a linear millimetre wave (mmWave) phased array antenna by considering the phase-only synthesis approach. For the first time, gradient boosting tree (GBT) is applied to estimate the phase values of a 16-element array antenna to generate different far-field radiation patterns. GBT predicts phases while the amplitude values have been equally set to generate different beam patterns for various 5G mmWave transmission scenarios such as multicast, unicast, broadcast and unmanned aerial vehicle (UAV) applications.
\end{abstract}

Index Terms-5G, phased array antenna, gradient boosting tree (GBT), machine learning (ML), millimetre wave (mmWave), array factor, phase-only synthesis.

\section{INTRODUCTION}

Uniformally excited array antennas synthesis namely amplitude-only array synthesis is a conventional method for antenna array optimization [1]. Amplitude-only array synthesis is useful for the sidelobe level (SLL) reduction by assigning different amplitude values for each array element. This method is mainly applicable for boresight far-field radiation pattern synthesis when the beam pattern starts to steer away from the boresight direction, amplitude weights need to be recalculated again to satisfy the SLL margin. However, it is also feasible to synthesize phased array antennas by phase tapering while element amplitudes are set to one. This approach is termed as phase-only array synthesis in the literature and has simpler feeding network compared to amplitude-only method. Basically, phase shifters are indispensable components of phased array systems irrespective of the synthesis technique while amplitude-only synthesis approach suffers from a complex feeding network due to tapering for each antenna element. Although phase-only approach is promising, there are only a few research studies dedicated to phase-only array synthesis due to the need for using non-linear optimization methods. For example, genetic algorithm (GA) approaches have been applied to the phase-only synthesis problems [2]-[4]. In addition, a non-linear phase-only synthesis GA-based approach was proposed in [5] to reduce the SLL for both planar and linear arrays. All of reported studies are based on optimisation techniques (mainly GA) and have several disadvantages including no-guarantee to converge or find the optimum solution, intractability of finding the optimal parameter settings, and the need to design a fitness function that largely impacts the final results. Here, for the first time machine learning (ML) is introduced to estimate the desired radiation patterns by applying the phase-only synthesis technique. Machine learning in contrast with optimisation models allows establishing a model describing multiple patterns that can be used to synthesise a similar pattern of interest. However, optimisation techniques must be run independently to estimate phases for each pattern of interest. As summary, one of significant advantages of ML methods is that they can learn from the data without any prior knowledge and the learned model can be used for future designs [6] .

On the other hand, recently there is a huge interest towards millimetre wave (mmWave) as an enabling technique for future 5G network [7]-[9]. Ofcom proposed $26 \mathrm{GHz}$ band as a pioneer band for $5 \mathrm{G}$ in Europe [10] while FCC proposed $28 \mathrm{GHz}$ band for 5G deployment in US [11]. In this paper a linear phased array antenna with 16 radiator elements has been considered to synthesis radiation pattern based on the phase-only approach. Gradient boosting tree (GBT) [12] is applied to estimate the phase values of each array elements to generate different array antenna far field patterns for various 26 GHz 5G applications; such as multicast, unicast, broadcast and Unmanned Aerial Vehicle (UAV) scenarios. GBT is based on training sequential weak learners by focusing on the residual error from the previous stage. Eventually, the final prediction is based on a vote from multiple learners. GBT is a powerful technique based on training multiple weak learners to improve the final performance and has lower sensitivity to outliers and has shown to perform well in various applications. As a result, it has been selected in this work.

\section{DATA GENERATION AND MODEL DESCRIPTION}

\section{A. Background}

The mmWave frequency bands suffer from high path loss due to their short wavelength. Array antenna provides an effective means of tackling this problem. Having an array results in narrow and sharp beams, and coverage can be extended by steering the beam in different directions. An array response is based on the array structure, separation distance between array elements, element weights and the excitation 
phases is called the array factor (AF) and, for a linear array with $M$ radiator elements, is calculated by

$$
\mathrm{AF}=\sum_{n=1}^{M} w_{n} \mathrm{e}^{j(n-1) \psi_{n}}
$$

where $w_{n}$ is an element weight, and $\psi_{n}$ is the excitation phase due to element position and observation direction. In this paper, we assume all elements have identical amplitudes $\forall(n \in M)\left[w_{n}=1\right]$ and only phase values are varying. To find the total far-field array pattern, AF is multiplied by the far-field radiation pattern of a single radiator element as follow:

$$
\text { Pattern Factor }(\mathrm{PF})=\text { Element Pattern }(\mathrm{EP}) \times \mathrm{AF}
$$

where pattern factor (PF) is an angular description of the far field radiation pattern of the whole array antenna considering the effect of EP and AF.

In this study, a linear array with 16 radiator elements ( $M$ $=16$ ) is considered. To perform the pattern factor, a single common patch antenna has been simulated and optimised to operate at $26 \mathrm{GHz}$, the prototype is fabricated and the measured radiation pattern is used as element pattern. 10000 PFs have been generated; half with the progressive phases and the second half with the random phases. Each PF represents an input and the corresponding phase elements as the output. Consequently, it covers the parameter space which are multiple possible phase elements as the output of the ML model and their corresponding PFs for the input to the learning algorithm.

\section{B. Gradient Boosting Tree}

Boosting methods are based on training sequential weak learners that performs only marginally better than the random guess. A small decision tree can be considered as an example.

Considering $N$ samples as $\left\{\left(\mathbf{x}_{1}, y_{1}\right),\left(\mathbf{x}_{2}, y_{2}\right), \ldots,\left(\mathbf{x}_{N}, y_{N}\right)\right\}$, at the start, same weights are assigned to them, e.g. $w_{1 i}=D$ where $\mathbf{x}, y_{i}$ and $w_{1 i}$ shows a data sample, its associated prediction, and the corresponding weight respectively. Nonetheless, in the following iteration, samples are weighted according to their miss-prediction at the previous step:

$$
\begin{aligned}
& \mathbf{p}_{k}=\mathbf{w}_{k} / \sum_{i=1}^{N} w_{k i} \\
& \mathbf{e}_{k}=\sum_{i=1}^{N} \mathbf{p}_{k}\left|\mathbf{h}_{k}\left(\mathbf{x}_{i}\right)-y_{i}\right| \\
& \mathbf{b}_{k}=\mathbf{e}_{k} /\left(1-\mathbf{e}_{k}\right) \\
& w_{(k+1) i}=w_{k i} \mathbf{b}_{k}^{1-\left|\mathbf{h}_{k}\left(\mathbf{x}_{i}\right)-y_{i}\right|}
\end{aligned}
$$

where $k$ is the iteration index, $\mathbf{e}_{k}$ is the error at step $k$ and $\mathbf{w}_{k+1}$ represents sample weights for the estimator at the next step. The procedure focuses on improving performance for mis-predicted samples by increasing their weights. Then, all estimators are combined by a weighted majority vote for the final prediction. This way of learning is less sensitive to outliers and noise in the data. GBT is a generalisation of boosting to an arbitrary differentiable loss function. For estimating multiple output a model for each target can be considered.

Considering $\mathbf{x}_{i}$ as a PF and $\mathbf{y}_{i}$ as $\psi_{i}$ with 16 phase values, GBT was used here to predict each $\psi_{n}$ for a given PF. In order to improve performance and let GBT focus on learning useful information, correlation between each desired PF and the whole data set was calculated. The threshold was considered as more than $90 \%$ and the data with lower correlation has been removed from the dataset. Then, the final dataset is the joint set of remained high correlated data to each desired PF. This step is only a preprocessing optional step.

After filtering based on the correlation, 6813 data points were considered for training (90\% of the remained data) and the remaining 757 points for testing the model. For each $\mathrm{PF}$, the input is represented by a vector of size 180 and the corresponding output is a vectors of size 16 representing 16 phase elements. The training and test accuracy were 0.001 and 0.03 respectively. Accuracy here is the normalised mean square error between the estimated 16 phases representing antenna elements and the true phases for PFs in the test or train sets.

\section{Phase Only Synthesis}

A main task of phase-only array synthesis is to determine the optimal set of values, $\psi_{n}$ in Eq. (1) to obtain the specific far-filed array pattern. Here, $16 \psi_{n}$ values are estimated to form different beam patterns to support various $5 \mathrm{G}$ mmWave applications.

\section{A. Unicast and Multicast Application}

It is predicted that the traffic demands continues to grow at an exponential rate in the the next-generation communication systems $(5 \mathrm{G})$. One of the aims of $5 \mathrm{G}$ communication is to provide improved services to existing $4 \mathrm{G}$ applications (multimedia streaming and High-QoE services) in addition to providing machine-type applications (smart environments and intelligent transport systems) as well as ultra-reliable low latency (URLLC) applications (autonomous vehicles, etc.).

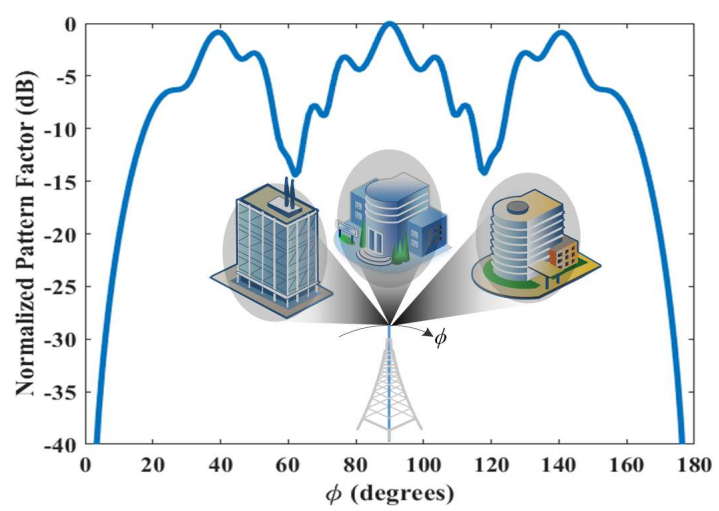

Fig. 1. Three beams normalised pattern factor based on estimated 16 phase values at $26 \mathrm{GHz}$. 


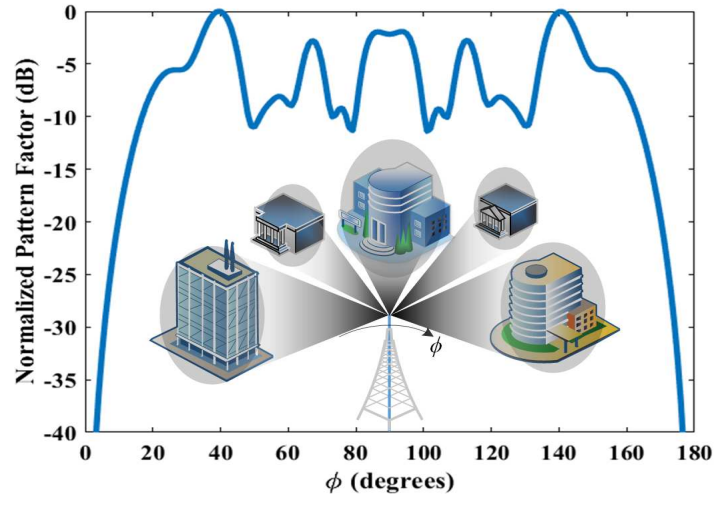

Fig. 2. Five-beam normalised pattern factor based on estimated 16 phase values at $26 \mathrm{GHz}$.

Furthermore, handling increasing demands for the unicast and multicast systems is significant in accessing broadband wireless services efficiently. Basically, sending information from a point in the network to another single point is called a unicast transmission which is the most common form of information transfer with easy and well-established techniques and protocols. In addition, multicast is described as having multiple users who have the subscription to the same service. Multicasting has the advantage of better use of the capacity in the cellular systems and is a practical solution for sharing information to a number of terminals. From the transmitter perspective, the difference between Unicast and Multicast is the number of RF chains to generate different independent data stream while for both applications multi-beam antenna is needed.

Phase difference determines the direction of propagation and amplitude distribution would control radiation pattern characteristics such as side-lobe lever (SLL), HPBW, and beam ripples. In the proposed method, we focus on only controlling the phase difference to come up with an aperture radiates toward the direction(s) of interest. To generate multiple beam patterns, fixed switched beam is one solution but it is costly and switching time between different fixed beams is an another issue that degrades the system performance. Also, using amplitude-only approach causes the gain reduction due to turning off some of radiator elements by tapering the amplitude. Here, phase-only synthesis is applied to determine the optimal set of phase values such that the array factor results in the multi beam pattern. To generate PF, which is defined in Eq. (2), a rectangular patch antenna at $26 \mathrm{GHz}$ is simulated and its radiation pattern is multiplied by the AF. To find 16 optimal phase values, first three-beam pattern is assigned as an input of the GBT model. Fig. 1 shows the normalized PF based on estimated phase values. In contrast to the GA approaches, once the data is trained with ML techniques, it can be used to predict the phase values for a new desired radiation pattern.

Next, five-beam pattern is applied as an input and phases are estimated to form the five-beam radiation pattern. Fig. 2 indicates five-beam radiation pattern suitable for multicast and unicast applications while the optimal phases are estimated by

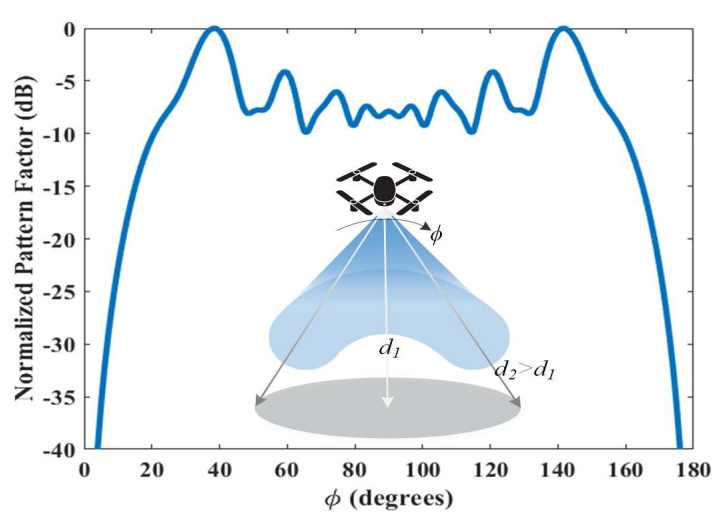

Fig. 3. Saddle-shaped beam pattern.

GBT. Considering more path loss in non-boresight direction in comparison to the boresight direction, five-beam pattern is generated by having higher beam gain in those angles far away form the Theta $=0^{\circ}$ to meet the link budget and have a same received power in all angles.

\section{B. Unmanned Aerial Vehicle (UAV) application}

Assuming a 3-D cone-shaped area as the illuminating region beneath the UAV, a circle-shaped contours of coverage is achieved on the earth [13]. Vertical distance from the vertex of the cone to the earth is clearly lower than that of any other non-vertical lines. From propagation point of view, the radiated waves through the vertical line experiences lower path loss in comparison with any other non-vertical lines and regions near the center point of the circle-shaped contours of coverage have higher EIRP values in comparison with the edge-regions, results in a non-uniform distribution of received power over the area of coverage. In order to have an almost uniform level of sensitivity over the entire area under coverage, the most appropriate shape is a saddle-shaped pattern in which the minimum gain pattern must be accrued at $(\theta=90)$ to reduce the EIRP of vertical line component while higher gain values are needed to compensate the higher path loss at the near-edge regions on the ground. Fig. 3 indicates the saddle-shaped array

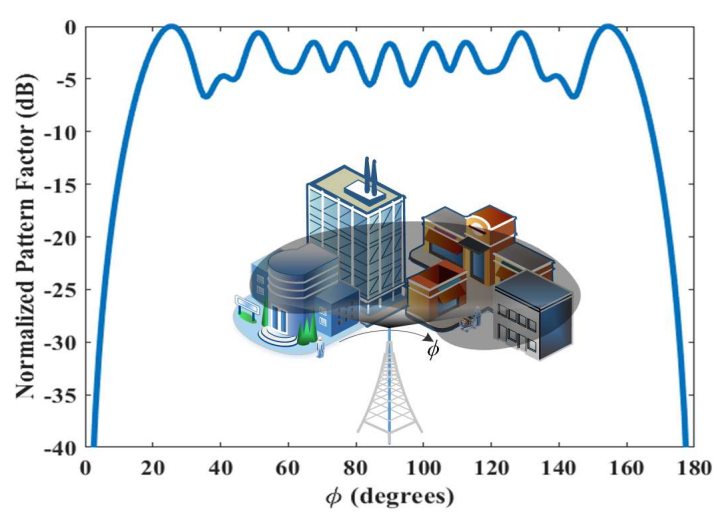

Fig. 4. Widebeam array pattern calculating for phase values predicted by GBT. 

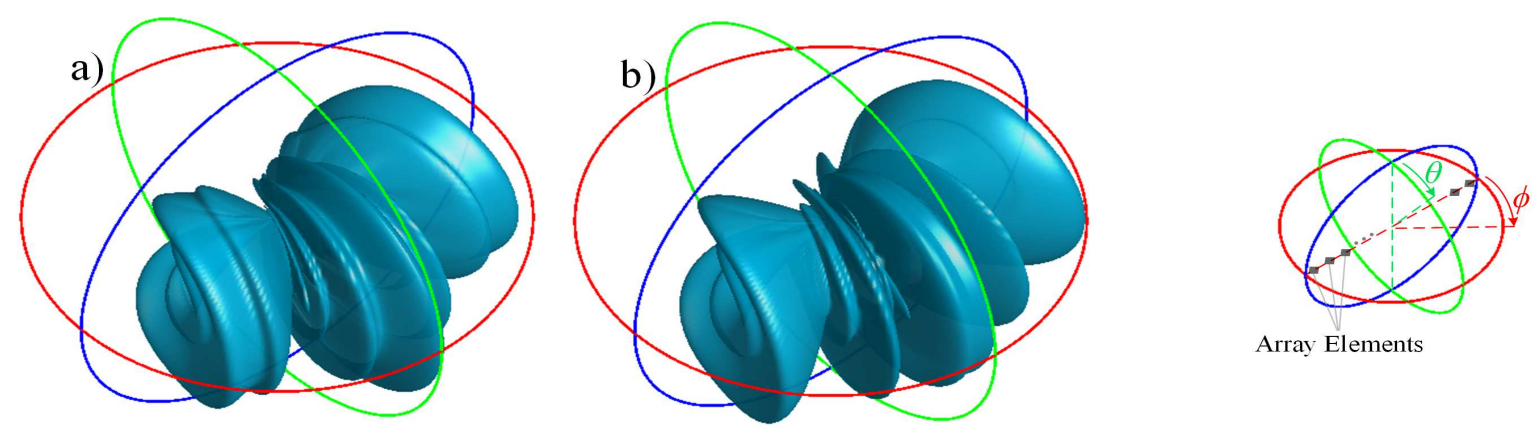

Array Elements
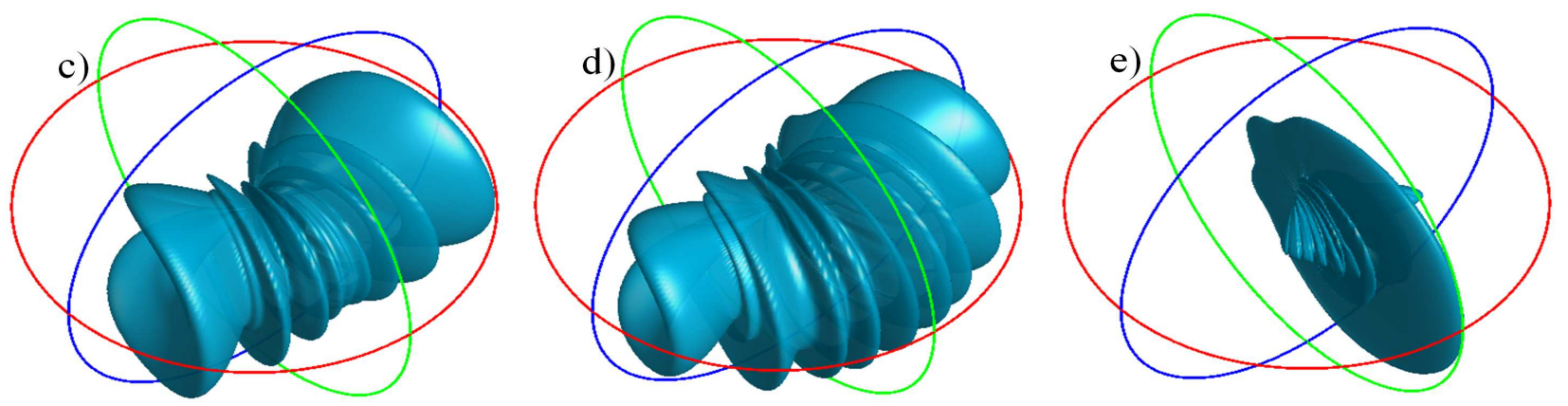

Fig. 5. 3D normalised radiation pattern factor in $\mathrm{dB}$ of (a) three-beam, (b) five-beam, (c) saddle-shaped beam, (d) widebeam, (e) boresight pattern for $\psi_{i}=0$ , and dynamic range of $60 \mathrm{~dB}$.

TABLE I

PREDICTED 16 PHASE VALUES FOR DIFFERENT RADIATION PATTERNS.

\begin{tabular}{c|cccc}
\hline Estimated Phases & Three-Beam Pattern & Five-Beam Pattern & Saddle-Shaped Beam Pattern & WideBeam Array Pattern \\
\hline$\psi_{1}$ & 0 & 0 & 0 & 0 \\
$\psi_{2}$ & 142.32847184 & 143.33452399 & 136.77605927 & 184.76658412 \\
$\psi_{3}$ & 281.99939286 & 282.62453362 & 273.55211855 & 309.52055325 \\
$\psi_{4}$ & 435.69848803 & 475.69848803 & 411.77337706 & 500.89647463 \\
$\psi_{5}$ & 562.63272243 & 561.77552221 & 547.10423709 & 645.04110651 \\
$\psi_{6}$ & 719.28436561 & 720.10224530 & 686.28896177 & 803.8512703 \\
$\psi_{7}$ & 401.60312986 & 891.60312986 & 883.54675412 & 1120.85281386 \\
$\psi_{8}$ & 436.89813568 & 496.89813568 & 439.64520644 & 666.78612754 \\
$\psi_{9}$ & 801.74963177 & 896.99513568 & 439.64520644 & 666.78612754 \\
$\psi_{10}$ & 719.32898648 & 720.11392745 & 883.54675412 & 1120.85281386 \\
$\psi_{11}$ & 562.87651446 & 561.63986522 & 686.28896177 & 803.8512703 \\
$\psi_{12}$ & 435.37910201 & 475.37910201 & 547.10423709 & 645.04110651 \\
$\psi_{13}$ & 281.42939179 & 282.91291166 & 411.77337706 & 500.89647463 \\
$\psi_{14}$ & 142.52491657 & 143.84732378 & 273.55211855 & 309.52055325 \\
$\psi_{15}$ & 0 & 0 & 136.77605927 & 0 \\
$\psi_{16}$ & & & 0 & 184.76658412 \\
\hline
\end{tabular}

pattern while the relative phase values are estimated by GBT.

\section{Broadcasting Application}

With broadcast transmissions, the same message is received by all users distributed in a spacious area, such as auditoriums, stadiums, shopping malls and working offices, the antenna with the semi-omnidirectional radiation is usually preferred. In addition to broadcast applications, a beam with wide beamwidth and stable gain is also very useful for the unicast beam training. Here, the aim is to estimate 16 phase values in such a way that widebeam pattern with stable gain would be achieved. By using GBT, 16 phase values are estimated and widebeam pattern is generated with stable gain over 100 degrees. Fig. 4 shows this widebeam pattern with slight variation in the gain.
Table I presents the estimated 16 phases for different scenarios. To ease understanding, 3D normalized PFs over $4 \pi$ steradians are presented in Fig. 5 in spherical coordinates. Each radiation pattern is normalized to its own maximum and Figs. 1 to 4 are the $2 \mathrm{D}$ views with $\theta=90,0<\phi<180$ cut of their respective 3Ds. Fig. 5 (a) and (b) are 3D view of the multicast/unicast scenario presented in Fig. 3 and 4, respectively. Fig. 5(c) is a saddle-shaped beam pattern which is the most suitable pattern for UAVs while Fig. 5(d) is widebeam pattern suitable for the broadcasting scenario. The radiation PF of the equally phase/amplitude distributed array is illustrated in Fig. 5(e) as a reference for comparison. 


\section{CONCLUSION}

A linear phased array antenna was synthesised by phaseonly approach with the ML technique. A $16 \times 1$ equally weighted-amplitude phased array antenna with half lambda element spacing was synthesized to obtain targeted beam patterns for different mmWave applications. For the first time multi-output GBT technique is applied to estimate 16 phase values to form different specific beam patterns. The phase tapering approach enable us to have a different beam patterns for various $5 \mathrm{G}$ use-case scenarios. It is worth mentioning that this method is applicable for all linear array geometry with arbitrary number of radiator elements.

\section{REFERENCES}

[1] K. Guney and M. Onay, "Amplitude-only pattern nulling of linear antenna arrays with the use of bees algorithm," Progress In Electromagnetics Research, vol. 70, pp. 21-36, 2007.

[2] Y. Liu, Y.-C. Jiao, Y.-M. Zhang, and Y.-Y. Tan, "Synthesis of phaseonly reconfigurable linear arrays using multiobjective invasive weed optimization based on decomposition," International Journal of Antennas and Propagation, vol. 2014, 2014.

[3] G. K. Mahanti, A. Chakraborty, and S. Das, "Phase-only and amplitudephase only synthesis of dual-beam pattern linear antenna arrays using floating-point genetic algorithms," Progress In Electromagnetics Research, vol. 68, pp. 247-259, 2007.

[4] G. Mahanti, A. Chakrabarty, and S. Das, "Discrete phase-only synthesis of a dual beam collinear dipole antenna array using genetic algorithms,' International Journal of Theoretical and Applied Computer Science, vol. 1, no. 1, pp. 63-70, 2006.

[5] J. F. DeFord and O. P. Gandhi, "Phase-only synthesis of minimum peak sidelobe patterns for linear and planar arrays," IEEE transactions on antennas and propagation, vol. 36, no. 2, pp. 191-201, 1988.

[6] M. Khalily, T. W. C. Brown, and R. Tafazolli, "Machine-learning-based approach for diffraction loss variation prediction by the human body," IEEE Antennas and Wireless Propagation Letters, vol. 18, no. 11, pp. 2301-2305, Nov 2019.

[7] M. Khalily, S. Taheri, S. Payami, M. Ghoraishi, and R. Tafazolli, "Indoor wideband directional millimeter wave channel measurements and analysis at $26 \mathrm{ghz}, 32 \mathrm{ghz}$, and $39 \mathrm{ghz}, "$ Transactions on Emerging Telecommunications Technologies, vol. 29, no. 10, p. e3311, 2018 , e3311 ett.3311. [Online]. Available: https://onlinelibrary.wiley.com/doi/ abs/10.1002/ett.3311

[8] M. M. Molu, P. Xiao, M. Khalily, K. Cumanan, L. Zhang, and R. Tafazolli, "Low-complexity and robust hybrid beamforming design for multi-antenna communication systems," IEEE Transactions on Wireless Communications, vol. 17, no. 3, pp. 1445-1459, March 2018.

[9] M. Khalily, R. Tafazolli, P. Xiao, and A. A. Kishk, "Broadband mmwave microstrip array antenna with improved radiation characteristics for different $5 \mathrm{~g}$ applications," IEEE Transactions on Antennas and Propagation, vol. 66, no. 9, pp. 4641-4647, Sep. 2018.

[10] Ofcom, "Ofcom notes: Update on 5G spectrum in the UK," 8 February 2017. [Online]. Available: https://www.ofcom.org.uk/_data/assets/pdf_ file/0021/97023/5G-update-08022017.pdf

[11] "FCC takes steps to facilitate mobile broadband and next generation wireless technologies in spectrum above 24 GHz," Federal Communications Commission, Jul 2016. [Online]. Available: https://www.fcc.gov/document/ fcc-adopts-rules-facilitate-next-generation-wireless-technologies

[12] J. H. Friedman, "Stochastic gradient boosting," Computational statistics \& data analysis, vol. 38, no. 4, pp. 367-378, 2002.

[13] A. Araghi, M. Mayiami, A. Montazeri, A. Foudazi, M. Yaghubi, and M. Bod, "Antenna selection procedure for bts over haps." Applied Computational Electromagnetics Society Journal, vol. 28, no. 9, 2013. 